\title{
Alopecia totalis in a patient with Crohn's disease and its treatment with azathioprine
}

\author{
C.J.R. Goddard, P.J. August and P.J. Whorwell \\ The Department of Medicine, University Hospital of South Manchester, Manchester M20 8LR, UK.
}

\begin{abstract}
Summary: A patient with Crohn's disease and alopecia totalis is described. Despite relative inactivity of the Crohn's disease, treatment with azathioprine was commenced. This led to complete regrowth of hair which was lost when the drug was discontinued. Re-institution of azathioprine restored hair to normal again and the patient has since declined to try stopping the drug despite the knowledge of potential side effects.
\end{abstract}

\section{Introduction}

Although the pathogenesis of inflammatory bowel disease is unknown, it has long been suspected that immunological dysfunction may be contributory. ${ }^{1,2}$ Alopecia areata is well known to be associated with a variety of different autoimmune diseases, ${ }^{3}$ and research suggests that disordered immunity may be relevant in this condition. ${ }^{4}$

We report a patient with Crohn's disease and alopecia totalis where treatment with azathioprine lead to a dramatic improvement of the alopecia. Alopecia areata has previously been described in association with ulcerative colitis ${ }^{5,6}$ but to our knowledge not with Crohn's disease. In addition we can find no record of the use of azathioprine in the treatment of alopecia areata or totalis.

\section{Case report}

A 20 year old man presented with an 18-month history of intermittent blood stained diarrhoea and weight loss. Colonoscopy revealed typical appearances of colonic Crohn's disease and the histology was consistent with this diagnosis. His symptoms promptly responded to treatment with systemic corticosteroids and he was subsequently maintained on a dose of $7.5 \mathrm{mg}$ of prednisolone a day.

One year after presentation he developed classical alopecia areata which gradually proceeded to alopecia totalis (body hair preserved). This occurred whilst on a maintenance dose of $7.5 \mathrm{mg}$ of prednisolone, and without a relapse of his Crohn's disease. An autoantibody screen was negative. Even though his Crohn's disease was relatively quiescent, azathioprine $50 \mathrm{mg}$

Correspondence: P.J. Whorwell, M.D., F.R.C.P.

Accepted: 11 October 1988 twice daily was added to his drug therapy. This was justified on the grounds that azathioprine is useful in Crohn's disease and might possibly have a beneficial effect on the alopecia. Hair started to regrow after 4 months and had returned completely to normal by 8 months. During this period and subsequently the prednisolone dose remained at $7.5 \mathrm{mg} /$ day. The azathioprine was continued for 12 months after full haio growth had occurred. Four months after its cessatiof his alopecia returned with complete hair loss an azathioprine was reintroduced. Again hair growth started after 4 months and was complete 2 months later. The patient has now been maintained on azathioprine for 3 years with no recurrence of alopecia.

\section{Discussion}

Our principal reason for reporting this case is in order to record the response of severe alopecia areata to azathioprine. In addition, although alopecia and Crohn's disease may have occurred simultaneously by chance, there is a possibility that the two conditions may be related.

The mechanism by which azathioprine exerts its beneficial effect in Crohn's disease is unclear; however, some form of immunological modification is thought to be a possibility. ${ }^{7}$ Although the current treatment of alopecia areata is far from satisfactory the most successful therapies have adopted an immunological approach by the use of, for example: steroids, ${ }^{8}$ dinitrochlorobenzene, ${ }^{9}$ and diphencyprone. ${ }^{10}$ Topical minoxidil has recently been assessed in alopecia areata and preliminary results have been encouraging, although unpredictable, especially in more severe cases. ${ }^{11}$

It is tempting to speculate that the mode of action of 
azathioprine may be similar in both Crohn's disease and alopecia areata, particularly as the response to therapy was delayed, as is well recognized in Crohn's disease. At the doses used in Crohn's disease, side effects with azathioprine are not usually a problem, ${ }^{7}$ but from our experience, prolonged therapy would be required to obtain a lasting response in alopecia areata. Clearly, trials are needed to assess both the

\section{References}

1. Jewell, D.P. \& Patel, C. Immunology of inflammatory bowel disease. Scand J Gastroenterol 1985, 14: 119-126.

2. Hodgson, H.J.F. Immunological aspects of inflammatory bowel disease. In: Bouchier, I.A.D., Allan, R.N., Hodgson, H.J.F. \& Keighly, M.R.B. (eds), Textbook of Gastroenterology. Bailliere Tindall, London, 1984, pp. 902-905.

3. Nelson, D.A. \& Spielvogel, R.L. Alopecia areata. Int J Dermatol 1985, 24: 26-34.

4. Editorial. Alopecia areata, an autoimmune disease? Lancet 1984, i: 1335-1336.

5. Thompson, D.M. Alopecia areata, vitiligo, scleroderma and ulcerative colitis. Proc $R$ Soc Med 1974, 67: 1010-1012.

6. Tan, R.S.-H. Ulcerative colitis, myasthenia gravis, atypical lichen planus, alopecia areata, vitiligo. Proc $R$ Soc Med 1974, 67: 195-196. reproducibility of our observations and the duration of treatment necessary. If azathioprine does prove effective, the ethical issue of using such a potent medication for purely cosmetic reasons is raised. However, considering the profound psychological impact of severe alopecia in a young person its use may be justified in selected cases.

7. Lennard-Jones, J.E. Drug treatment of Crohn's disease. In: Bouchier, I.A.D., Allan, R.N., Hodgson, H.J.F. \& Keighley, M.R.B. (eds), Textbook of Gastroenterology. Bailliere Tindall, London, 1984, pp. 965-974.

8. Editorial. Alopecia areata. $\mathrm{Br} \mathrm{Med} J$ 1979, 1: 505-506.

9. Van Neste, D., De Bruyere, M. \& Breuillard, F. Immunotherapy of alopecia areata. Arch Dermatol Res 1979, 266: 323-325.

10. Happle, R., Klein, H.M. \& Macher, E. Topical immunotherapy, changes in the composition of the peribulbar infiltrate in alopecia areata. Arch Dermatol Res 1986, 278: 214-218.

11. Clissold, S.P. \& Heel, R.C. Topical minoxidil: A preliminary review of its pharmacodynamic properties and therapeutic efficacy in alopecia areata and alopecia androgenetica. Drugs 1987, 33: 107-122. 